\title{
Limit Theorems for a Storage Process with a Random Release Rule
}

\author{
Lakhdar Meziani \\ Department of Mathematics, Faculty of Science, King Abdulaziz University, Jeddah, Saudi Arabia \\ Email: mezianilakhdar@hotmail.com
}

Received August 29, 2012; revised October 8, 2012; accepted October 15, 2012

\begin{abstract}
We consider a discrete time Storage Process $X_{n}$ with a simple random walk input $S_{n}$ and a random release rule given by a family $\left\{U_{x}, x \geq 0\right\}$ of random variables whose probability laws $\left\{\mu_{x}, x \geq 0\right\}$ form a convolution semigroup of measures, that is, $\mu_{x} \times \mu_{y}=\mu_{x+y}$. The process $X_{n}$ obeys the equation:

$X_{0}=0, U_{0}=0, X_{n}=S_{n}-U_{S_{n}}, n \geq 1$. Under mild assumptions, we prove that the processes $U_{S_{n}}$ and $X_{n}$ are simple random walks and derive a SLLN and a CLT for each of them.
\end{abstract}

Keywords: Storage Process; Random Walk; Strong Law of Large Numbers; Central Limit Theorem

\section{Introduction and Assumptions}

The formal structure of a general storage process displays two main parts: the input process and the release rule. The input process, mostly a compound Poisson process $A(t)$, describes the material entering in the system during the interval $[0, t]$. The release rule is usually given by a function $r(x)$ representing the rate at which material flows out of the system when its content is $x$. So the state $X(t)$ of the system at time $t$ obeys the well known equation:

$X(t)=X(0)+A(t)-\int_{0}^{t} r(X(s)) \mathrm{d} s$.

Limit theorems and approximation results have been obtained for the process $X(t)$ by several authors, see [1-5] and the references therein. In this paper we study a discrete time new storage process with a simple random walk input $S_{n}$ and a random release rule given by a family of random variables $\left\{U_{x}, x \geq 0\right\}$, where $U_{x}$ has to be interpreted as the amount of material removed when the state of the system is $x$. Hence the evolution of the system obeys the following equation: $X_{0}=0$, $U_{0}=0, \quad X_{n}=S_{n}-U_{S_{n}}, \quad n \geq 1$. where $S_{0}=0$, $S_{n}=Z_{1}+Z_{2}+\cdots+Z_{n}$, for i.i.d. positive random variables $Z_{n}$, with $E\left(Z_{1}\right)=\alpha>0$, and $\sigma_{Z_{1}}^{2}=\sigma^{2}<\infty$.

We will make the following assumptions:

1.1. The probability distributions $\left\{\mu_{x}, x \geq 0\right\}$ of the random variables $\left\{U_{x}, x \geq 0\right\}$ form a convolution semigroup of measures:

$$
\forall x, y \geq 0, \mu_{x} \times \mu_{y}=\mu_{x+y},
$$

We will assume that for each $x, \mu_{x}$ is supported by the interval $[0, x]$, that is, $\forall x \in \mathrm{R}_{+}, \mu_{x}[0, x]=1$. Consequently, for $x \leq y$ the distribution of $U_{y}-U_{x}$ is the same as that of $U_{y-x}$, (see 2.2 (ii)).

1.2. Also we will need some smoothness properties for the stochastic process $U_{x}, x \geq 0$. These will be achieved if we impose the following continuity condition:

$$
\lim _{x \rightarrow 0_{+}} \mu_{x}=\delta_{0}
$$

where $\delta_{0}$ is the unit mass at 0 and the limit is in the sense of the weak convergence of measures.

1.3. The two families of random variables $\left\{U_{x}, x \geq 0\right\}$ and $\left\{Z_{n}, n \geq 1\right\}$ are independent.

\section{Construction of the Processes}

$$
\left\{Z_{n}, \boldsymbol{n} \geq \mathbf{1}\right\},\left\{\boldsymbol{U}_{\boldsymbol{x}}, \boldsymbol{x} \geq \mathbf{0}\right\} \text { and }\left\{\boldsymbol{X}_{n}, \boldsymbol{n} \geq \mathbf{0}\right\}
$$

2.1. Let $\lambda$ be a probability measure on the Borel sets $\mathbf{B}_{\mathrm{R}_{+}}$of the positive real line $R_{+}$and form the infinite product space $\left(\Omega_{1}, \mathbf{F}_{1}, P_{1}\right)=\left(\mathrm{R}_{+}^{\mathrm{N}}, \mathbf{B}_{\mathrm{R}_{+}^{\mathrm{N}}}, \lambda^{\otimes \mathrm{N}}\right)$. Now, as usual define random variables $Z_{n}$ on $\Omega_{1}$ by:

$$
Z_{n}\left(\omega_{1}\right)=\omega_{1}(n) \text {, if } \omega_{1}=\left(\omega_{1}(k)\right)_{k} \in \Omega_{1} .
$$

Then the $Z_{n}$ are independent identically distributed with common distribution $\lambda$ We will assume that $E\left(Z_{1}\right)=\alpha>0$, and $\sigma_{Z_{1}}^{2}=\sigma^{2}<\infty$.

2.2. Let $\left\{\mu_{x}, x \geq 0\right\}$ be a semigroup of convolution of probability measures on $\mathbf{R}_{+}, \mathbf{B}_{\mathrm{R}_{+}}$with $\mu_{0}=\delta_{0}$ and satisfying (1.2) then, it is well known, that there is a probability space $\left(\Omega_{2}, \mathbf{F}_{2}, P_{2}\right)$ and a family 
$\left\{U_{x}, x \geq 0\right\}$ of positive random variables defined on this space such that the following properties hold:

(i). Under $P_{2}$ the distribution of $U_{x}$ is $\mu_{x}$, $U_{0}=0$.

(ii). For $x \leq y$, the random variables $U_{y}-U_{x}$ and $U_{y-x}$ have under $P_{2}$ the same distribution $\mu_{y-x}$.

(iii). For every $0 \leq x_{1} \leq x_{2} \leq \cdots \leq x_{n}$, the increments $U_{x_{1}}, U_{x_{2}}-U_{x_{1}}, \cdots, U_{x_{n}}-U_{x_{n-1}}$ are independent.

(iv) . For almost all $\omega_{2} \in \Omega_{2}$ the function

$x \rightarrow U_{x}\left(\omega_{2}\right)$ is right continuous with left hand limit (cadlag).

From (iv) we deduce:

$(v)$. The function $\left(x, \omega_{2}\right) \rightarrow U_{x}\left(\omega_{2}\right)$ is measurable on the product space $R_{+} \times \Omega_{2}$.

2.3. The basic probability space for the storage process $X_{n}$ will be the product

$(\Omega, \mathbf{F}, P)=\left(\Omega_{1} \times \Omega_{2}, \mathbf{F}_{1} \otimes \mathbf{F}_{2}, P_{1} \otimes P_{2}\right)$. Then we define $X_{n}$ by the following recipe:

$$
X_{0}=0 \text {, }
$$

$X_{n}(\omega)=S_{n}\left(\omega_{1}\right)-U_{S_{n}\left(\omega_{1}\right)}\left(\omega_{2}\right), \quad$ if $\quad \omega=\left(\omega_{1}, \omega_{2}\right) \in \Omega$, $n \geq 1$. where $S_{n}$ is the simple random walk with: $S_{0}=0, \quad S_{n}=Z_{1}+Z_{2}+\cdots+Z_{n}, \quad n \geq 1$.

2.4. Since $S_{n}$ is a simple random walk, the random variables $S_{n}-S_{k}$ and $S_{n-k}$ have the same distribution for $k \leq n$.

\section{The Main Results}

The main objective is to establish limit theorems for the processes $U_{S_{n}}$ and $X_{n}$. Since the behavior of $S_{n}$ is well understood, we will focus attention on the structure of the process $U_{S_{n}}$. The outstanding fact is that $U_{S_{n}}$ itself is a simple random walk. First we need some preparation.

3.1. Proposition: For every measurable bounded function $f: \mathbf{R}_{+} \rightarrow \mathrm{R}$, the function

$x \rightarrow \mu_{x}(f)=\int_{\mathrm{R}_{+}} f(t) \mu_{x}(\mathrm{~d} t)$ is measurable. Thus for any Borel set $A$ of $\mathrm{R}_{+}$the function $x \rightarrow \mu_{x}(A)$ is measurable.

Proof: Assume first $f: \mathrm{R}_{+} \rightarrow \mathrm{R}$ continuous and bounded, then from (1.2) we have

$$
\lim _{x \rightarrow 0_{+}} \mu_{x}(f)=\delta_{0}(f)=f(0) .
$$

Now by (1.1) we have

$$
\begin{aligned}
& \mu_{x+y}(f)=\int_{\mathrm{R}_{+}} f(t) \cdot \mu_{x} \times \mu_{y}(\mathrm{~d} t) \\
& =\int_{\mathrm{R}_{+} \times \mathrm{R}_{+}} f(t+s) \mu_{x}(\mathrm{~d} t) \cdot \mu_{y}(\mathrm{~d} s) \\
& \rightarrow \int_{\mathrm{R}_{+}} f(t) \mu_{x}(\mathrm{~d} t), y \downarrow 0 .
\end{aligned}
$$

by (1.2) and the bounded convergence theorem. Consequently the function $x \rightarrow \mu_{x}(f)=\int_{\mathrm{R}_{+}} f(t) \mu_{x}(\mathrm{~d} t)$ is right continuous for all $x \geq 0$, hence it is measurable if $f$ is continuous and bounded. Next consider the class of functions:

$$
\mathbf{H}=\left\{\begin{array}{l}
f: \mathrm{R}_{+} \rightarrow \mathrm{R}, \text { such that the function } \\
x \rightarrow \mu_{x}(f)=\int_{\mathrm{R}_{+}} f(t) \mu_{x}(\mathrm{~d} t) \text {, is measurable. }
\end{array}\right\}
$$

then $\mathbf{H}$ is a vector space satisfying the conditions of Theorem I,T20 in [6]. Moreover, by what just proved, H contains the continuous bounded functions

$f: \mathbf{R}_{+} \rightarrow \mathrm{R}$, therefore $\mathbf{H}$ contains every measurable bounded function $f: \mathrm{R}_{+} \rightarrow \mathrm{R}$,

3.2. Remark: Let $E_{P_{1}}, E_{P_{2}}, E_{P}$, be the expectation operators with respect to $P_{1}, P_{2}, P$, respectively. Since $P=P_{1} \otimes P_{2}$, we have $E_{P_{1}} \cdot E_{P_{2}}=E_{P_{2}} \cdot E_{P_{1}}$, by Fubini theorem.

3.3. Proposition: Let $Y$ be a positive random variable on $\left(\Omega_{1}, \mathbf{F}_{1}, P_{1}\right)$ with probability distribution $\gamma$. Then the function $U_{Y}$ defined on $(\Omega, \mathbf{F}, P)$ by:

$$
\omega=\left(\omega_{1}, \omega_{2}\right) \rightarrow U_{Y}(\omega)=U_{Y\left(\omega_{1}\right)}\left(\omega_{2}\right)
$$

is a random variable such that

$$
E_{P}\left(f\left(U_{Y}\right)\right)=\int_{\mathrm{R}_{+} \times \mathrm{R}_{+}} f(t) \mu_{y}(\mathrm{~d} t) \cdot \gamma(\mathrm{d} y)
$$

for every measurable positive function $f: \mathbf{R}_{+} \rightarrow \mathrm{R}$. In particular the probability distribution of $U_{Y}$ is given by:

$$
\begin{gathered}
A \in \mathbf{B}_{\mathrm{R}_{+}} \\
P\left(U_{Y} \in A\right)=\int_{\mathrm{R}_{+}} \mu_{y}(A) \gamma(\mathrm{d} y)
\end{gathered}
$$

and its expectation is equal to

$$
E_{P}\left(U_{Y}\right)=\int_{\mathrm{R}_{+} \times \mathrm{R}_{+}} t \mu_{y}(\mathrm{~d} t) \cdot \gamma(\mathrm{d} y)
$$

Proof: Define $T: \Omega_{1} \times \Omega_{2} \rightarrow \mathrm{R}_{+} \times \Omega_{2}$ by $T\left(\omega_{1}, \omega_{2}\right)=\left(Y\left(\omega_{1}\right), \omega_{2}\right)$ and $S: \mathrm{R}_{+} \times \Omega_{2} \rightarrow \mathrm{R}_{+}$by $S\left(x, \omega_{2}\right)=U_{x}\left(\omega_{2}\right)$. It is clear that $T$ is measurable. Also $S$ is measurable by $2.2(v)$, so $S \circ T=U_{Y}$ is measurable.

(3.4) is a simple change of variable formula since $E_{P}=E_{P_{1}} \cdot E_{P_{2}}$.

3.7. Proposition: For all $1 \leq k \leq n$, the random variables $U_{S_{n}}-U_{S_{k}}, U_{S_{n}-S_{k}}, U_{S_{n-k}}$ have the same probability distribution.

Proof: It is enough to show that for every positive measurable function $f: \mathbf{R}_{+} \rightarrow \mathbf{R}$, we have:

$$
\begin{aligned}
& E_{P}\left(f\left(U_{S_{n}}-U_{S_{k}}\right)\right) \\
& =E_{P}\left(f\left(U_{S_{n}-S_{k}}\right)\right)=E_{P}\left(f\left(U_{S_{n-k}}\right)\right) .
\end{aligned}
$$


Since $E_{P}=E_{P_{1}} \cdot E_{P_{2}}$, we can write:

$$
\begin{aligned}
& E_{P}\left(f\left(U_{S_{n}}-U_{S_{k}}\right)\right) \\
& =\iint_{\Omega_{1} \Omega_{2}} f\left(U_{S_{n}\left(\omega_{1}\right)}\left(\omega_{2}\right)-U_{S_{k}\left(\omega_{1}\right)}\left(\omega_{2}\right)\right) P_{1}\left(\mathrm{~d} \omega_{1}\right) \cdot P_{2}\left(\mathrm{~d} \omega_{2}\right) .
\end{aligned}
$$

But for each fixed $\omega_{1} \in \Omega_{1}$ we get from 2.2(ii):

$$
\begin{aligned}
& \int_{\Omega_{2}} f\left(U_{S_{n}\left(\omega_{1}\right)}\left(\omega_{2}\right)-U_{S_{k}\left(\omega_{1}\right)}\left(\omega_{2}\right)\right) P_{2}\left(\mathrm{~d} \omega_{2}\right) \\
& =\int_{\Omega_{2}} f\left(U_{S_{n}\left(\omega_{1}\right)-S_{k}\left(\omega_{1}\right)}\left(\omega_{2}\right)\right) P_{2}\left(\mathrm{~d} \omega_{2}\right) \\
& =\mu_{S_{n}\left(\omega_{1}\right)-S_{k}\left(\omega_{1}\right)}(f)
\end{aligned}
$$

Applying $E_{P_{1}}$ to both sides of this formula we get the first equality of (3.7). To get the second one, observe that the function $\omega_{1} \rightarrow \mu_{S_{n}\left(\omega_{1}\right)-S_{k}\left(\omega_{1}\right)}(f)$ is measurable (Proposition 3.1) and use the fact that under $P_{1}$, the random variables $S_{n}-S_{k}$ and $S_{n-k}$ have the same probability distribution by 2.4 .

3.8. Theorem: The process $U_{S_{n}}, n \geq 0$ is a simple random walk with:

$U_{S_{0}}=U_{0}=0$

and $P\left(U_{S_{1}} \in A\right)=P\left(U_{Z_{1}} \in A\right)=\int_{R_{+}} \mu_{z}(A) \lambda(\mathrm{d} z)$

Proof: We prove that for all integers $1 \leq i \leq j \leq k \leq n$, and all positive measurable functions $f, g, h: \mathrm{R}_{+} \rightarrow \mathrm{R}$ we have:

$$
\begin{aligned}
& E_{P}\left(f\left(U_{S_{n}}-U_{S_{k}}\right) \cdot g\left(U_{S_{k}}-U_{S_{j}}\right) \cdot h\left(U_{S_{j}}-U_{S_{i}}\right)\right) \\
= & E_{P} f\left(U_{S_{n}}-U_{S_{k}}\right) \cdot E_{P}\left(g\left(U_{S_{k}}-U_{S_{j}}\right)\right) \\
& E_{P}\left(h\left(U_{S_{j}}-U_{S_{i}}\right)\right)
\end{aligned}
$$

Let $\omega_{1}$ be fixed in $\Omega_{1}$. By 2.2 (ii),(iii), under $P_{2}$ the random variables

$$
\begin{aligned}
& U_{S_{n}\left(\omega_{1}\right)}-U_{S_{k}\left(\omega_{1}\right)}, U_{S_{k}\left(\omega_{1}\right)}-U_{S_{j}\left(\omega_{1}\right)}, \\
& U_{S_{j}\left(\omega_{1}\right)}-U_{S_{i}\left(\omega_{1}\right)},
\end{aligned}
$$

are independent. Therefore, applying first $E_{P_{2}}$ in the L.H.S of (3.8), we get the formula:

$$
\begin{aligned}
& E_{P_{2}}\left(\left(f\left(U_{S_{n}\left(\omega_{1}\right)}-U_{S_{k}\left(\omega_{1}\right)}\right)\right) \cdot\left(g\left(U_{S_{k}\left(\omega_{1}\right)}-U_{S_{j}\left(\omega_{1}\right)}\right)\right)\right. \\
\cdot & \left.h\left(U_{S_{j}\left(\omega_{1}\right)}-U_{S_{i}\left(\omega_{1}\right)}\right)\right) \\
= & E_{P_{2}}\left(f\left(U_{S_{n}\left(\omega_{1}\right)}-U_{S_{k}\left(\omega_{1}\right)}\right)\right) \cdot E_{P_{2}}\left(g\left(U_{S_{k}\left(\omega_{1}\right)}-U_{S_{j}\left(\omega_{1}\right)}\right)\right) \\
\cdot & E_{P_{2}}\left(h\left(U_{S_{j}\left(\omega_{1}\right)}-U_{S_{i}\left(\omega_{1}\right)}\right)\right)
\end{aligned}
$$

But $\quad U_{S_{n}\left(\omega_{1}\right)}-U_{S_{k}\left(\omega_{1}\right)}, U_{S_{k}\left(\omega_{1}\right)}-U_{S_{j}\left(\omega_{1}\right)}, U_{S_{j}\left(\omega_{1}\right)}-U_{S_{i}\left(\omega_{1}\right)}$ have distributions $\mu_{S_{n}\left(\omega_{1}\right)-S_{k}\left(\omega_{1}\right)}, \quad \mu_{S_{k}\left(\omega_{1}\right)-S_{j}\left(\omega_{1}\right)}$,
$\mu_{S_{j}\left(\omega_{1}\right)-S_{i}\left(\omega_{1}\right)}$, respectively. Thus:

$$
\begin{aligned}
& E_{P_{2}}\left(f\left(U_{S_{n}\left(\omega_{1}\right)}-U_{S_{k}\left(\omega_{1}\right)}\right)\right)=\mu_{S_{n}\left(\omega_{1}\right)-S_{k}\left(\omega_{1}\right)}(f) \\
& E_{P_{2}}\left(g\left(U_{S_{k}\left(\omega_{1}\right)}-U_{S_{j}\left(\omega_{1}\right)}\right)\right)=\mu_{S_{k}\left(\omega_{1}\right)-S_{j}\left(\omega_{1}\right)}(g) \\
& E_{P_{2}}\left(h\left(U_{S_{j}\left(\omega_{1}\right)}-U_{S_{i}\left(\omega_{1}\right)}\right)\right)=\mu_{S_{j}\left(\omega_{1}\right)-S_{i}\left(\omega_{1}\right)}(h)
\end{aligned}
$$

By Proposition 3.1, the R.H.S of these equalities are random variables of $\omega_{1}$, independent under $P_{1}$ since they are measurable functions of the independent random variables $S_{n}-S_{k}, \quad S_{k}-S_{j}, \quad S_{j}-S_{i}$. Therefore, applying $E_{P_{1}}$ to both sides of formula (*) we get the proof of (3.8):

$$
\begin{aligned}
& E_{P_{1}} E_{P_{2}}\left(\left[f\left(U_{S_{n}}-U_{S_{k}}\right) \cdot g\left(U_{S_{k}}-U_{S_{j}}\right) \cdot h\left(U_{S_{j}}-U_{S_{i}}\right)\right]\right) \\
& =E_{P_{1}}\left[E_{P_{2}}\left(f\left(U_{S_{n}\left(\omega_{1}\right)}-U_{S_{k}\left(\omega_{1}\right)}\right)\right)\right. \\
& \left.\cdot E_{P_{2}}\left(g\left(U_{S_{k}\left(\omega_{1}\right)}-U_{S_{j}\left(\omega_{1}\right)}\right)\right) \cdot E_{P_{2}}\left(h\left(U_{S_{j}\left(\omega_{1}\right)}-U_{S_{i}\left(\omega_{1}\right)}\right)\right)\right] \\
& E_{P_{1}} E_{P_{2}}\left(f\left(U_{S_{n}\left(\omega_{1}\right)}-U_{S_{k}\left(\omega_{1}\right)}\right)\right) \\
& \cdot E_{P_{1}} E_{P_{2}}\left(g\left(U_{S_{k}\left(\omega_{1}\right)}-U_{S_{j}\left(\omega_{1}\right)}\right)\right) \\
& \cdot E_{P_{1}} E_{P_{2}}\left(h\left(U_{S_{j}\left(\omega_{1}\right)}-U_{S_{i}\left(\omega_{1}\right)}\right)\right) \\
& =E_{P}\left(f\left(U_{S_{n}}-U_{S_{k}}\right)\right) \cdot E_{P}\left(g\left(U_{S_{k}}-U_{S_{j}}\right)\right) \\
& \cdot E_{P}\left(h\left(U_{S_{j}}-U_{S_{i}}\right)\right) .
\end{aligned}
$$

To achieve the proof, write $U_{S_{n}}$ as follows: $U_{S_{n}}=\sum_{1}^{n}\left(U_{S_{k}}-U_{S_{k-1}}\right)$, where the $U_{S_{k}}-U_{S_{k-1}}$ are independent with the same distribution given by

$$
P\left(U_{Z_{k}} \in A\right)=\int_{R_{+}} \mu_{z}(A) \lambda(\mathrm{d} z)
$$

according to (3.5).

3.9. Proposition: For every positive measurable function $f: \mathbf{R}_{+} \rightarrow \mathbf{R}$, we have:

$$
E_{P}\left(f\left(U_{S_{n}}\right)\right)=\int_{\mathrm{R}_{+} \times \mathrm{R}_{+}} f(t) \cdot \mu_{s}(\mathrm{~d} t) \cdot \lambda^{* n}(\mathrm{~d} s)
$$

$\lambda^{* n}$ being the $\mathrm{n}$-fold convolution of the probability $\lambda$. In particular the distribution law of the process $U_{S_{n}}$ is given by:

$$
B \in \mathbf{B}_{\mathrm{R}_{+}}, \quad P\left(U_{S_{n}} \in B\right)=\int_{\mathrm{R}_{+}} \mu_{s}(B) \lambda^{* n}(\mathrm{~d} s)
$$

and its expectation is:

$$
E_{P}\left(U_{S_{n}}\right)=\int_{\mathrm{R}_{+} \times \mathrm{R}_{+}} t \mu_{s}(\mathrm{~d} t) \cdot \lambda^{* n}(\mathrm{~d} s)
$$


Proof: We have:

$$
\begin{aligned}
E_{P}\left(f\left(U_{S_{n}}\right)\right) & =E_{P_{1}} E_{P_{2}}\left(f\left(U_{S_{n}\left(\omega_{1}\right)}\left(\omega_{2}\right)\right)\right) \\
& =E_{P_{1}} \int_{R_{+}} f(t) \mu_{S_{n}\left(\omega_{1}\right)}(\mathrm{d} t)
\end{aligned}
$$

and, by Proposition 3.1, the function

$\omega_{1} \rightarrow \int_{R_{+}} f(t) \mu_{S_{n}\left(\omega_{1}\right)}(\mathrm{d} t)$ is a measurable function of $S_{n}\left(\omega_{1}\right)$. Since $S_{n}=Z_{1}+Z_{2}+\cdots+Z_{n}$ is a simple random walk with the $Z_{n}$ having distribution $\lambda$, the random variable $S_{n}$ has the distribution $\lambda^{* n}$. So, by a simple change of variable we get:

$E_{P_{1}} \int_{R_{+}} f(t) \mu_{S_{n}\left(\omega_{1}\right)}(\mathrm{d} t)=\int_{R_{+} R_{+}} f(t) \mu_{s}(\mathrm{~d} t) \cdot \lambda^{* n}(\mathrm{~d} s)$. So formula (3.9) is proved. To get the distribution law of the process $U_{S_{n}}$, take $f$ equal to the characteristic function of some Borel set B.

3.10. Remark: Let $v$ be the distribution of $U_{Z_{1}}$, that is $v(A)=\int_{\mathrm{R}_{+}} \mu_{z}(A) \lambda(\mathrm{d} z)$ and let

$\beta=E_{P}\left(U_{Z_{1}}\right)=\int_{R_{+} \times R_{+}} t \mu_{z}(\mathrm{~d} t) \cdot \lambda(\mathrm{d} z)$, then as a direct consequence of theorem $\mathbf{3 . 8}$,

$$
\begin{aligned}
& P\left(U_{S_{n}} \in B\right)=v^{* n}(B) \\
& E_{P}\left(U_{S_{n}}\right)=n \cdot \beta
\end{aligned}
$$

Now we turn to the structure of the process $X_{n}$. We need the following technical lemma:

3.11. Lemma: For every Borel positive function

$F: \mathrm{R}_{+} \times \mathrm{R}_{+} \rightarrow \mathrm{R}$, the function $\varphi: s \rightarrow \int_{\mathrm{R}_{+}} F(s, t) \mu_{s}(\mathrm{~d} t)$ is measurable.

Proof: Start with $F=I_{A \times B}$, the characteristic function of the measurable rectangle $A \times B$, in which case we have $\varphi(s)=I_{A}(s) \mu_{s}(B)$. Since by proposition 3.1, the function $s \rightarrow \mu_{s}(B)$ is measurable we deduce that $\varphi$ is measurable in this case. Next consider the family

$\Gamma=\left\{B \in \mathbf{B}_{\mathrm{R}_{+} \times \mathrm{R}_{+}}: s \rightarrow \int_{\mathrm{R}_{+}} I_{B}(s, t) \mu_{s}(\mathrm{~d} t)\right.$, is measurable. $\}$

It is easy to check that $\Gamma$ is a monotone class closed under finite disjoint unions. Since it contains the measurable rectangles, we deduce that $\Gamma=\mathbf{B}_{\mathrm{R}_{+} \times R_{+}}$. Finally consider the following class of Borel positive functions

$\mathbf{P}=\left\{F: \mathrm{R}_{+} \times \mathrm{R}_{+} \rightarrow \mathrm{R}, \varphi(s)=\int_{\mathrm{R}_{+}} F(s, t) \mu_{s}(\mathrm{~d} t)\right.$ is Borel $\}$

It is clear that $\mathbf{P}$ is closed under addition and, by the step above, it contains the simple Borel positive functions. By the monotone convergence theorem, $\mathbf{P}$ is ex- actly the class of all Borel positive functions.

3.12. Theorem: The random variables

$Z_{k}-\left(U_{S_{k}}-U_{S_{k-1}}\right), k=1,2, \cdots$, are independent with the same distribution given by: for $B \in \mathbf{B}_{\mathrm{R}_{+}}$

$$
\begin{aligned}
& P\left(\left(Z_{k}-\left(U_{S_{k}}-U_{S_{k-1}}\right)\right) \in B\right) \\
& =\int_{\mathrm{R}_{+}} \mu_{s}(s-B) \cdot \lambda(\mathrm{d} s)
\end{aligned}
$$

Consequently the storage process

$X_{n}=S_{n}-U_{S_{n}}=\sum_{1}^{n}\left(Z_{k}-\left(U_{S_{k}}-U_{S_{k-1}}\right)\right)$, is a simple random walk with the basic distribution (3.12).

Proof: For each integer $r \geq 0$, and each $\left(\omega_{1}, \omega_{2}\right) \in \Omega_{1} \times \Omega_{2}$, put:

$$
\begin{aligned}
& W_{r}\left(\omega_{1}, \omega_{2}\right) \\
& =Z_{r}\left(\omega_{1}\right)-\left(U_{S_{r}\left(\omega_{1}\right)}\left(\omega_{2}\right)-U_{S_{r-1}\left(\omega_{1}\right)}\left(\omega_{2}\right)\right)
\end{aligned}
$$

So it is enough to prove that for all $0 \leq i \leq j \leq k$ and all Borel positive functions $f, g, h: \mathbf{R}_{+} \rightarrow \mathrm{R}$, we have:

$$
\begin{aligned}
& E_{P}\left(f\left(W_{k}\right) \cdot g\left(W_{j}\right) \cdot h\left(W_{i}\right)\right) \\
& =E_{P}\left(f\left(W_{k}\right)\right) \cdot E_{P}\left(g\left(W_{j}\right)\right) \cdot E_{P}\left(h\left(W_{i}\right)\right)
\end{aligned}
$$

From the construction of the process $U_{S_{n}}$, we know that for $\omega_{1}$ fixed, the random variables $W_{r}\left(\omega_{1}, \omega_{2}\right)$, $r=i, j, k$, are independent under $P_{2}$ (see 2.2 (iii)). So, applying $E_{P_{2}}$ to $f\left(W_{k}\right) \cdot g\left(W_{j}\right) \cdot h\left(W_{i}\right)$, we get:

$$
\begin{aligned}
& E_{P_{2}}\left(f\left(W_{k}\right) \cdot g\left(W_{j}\right) \cdot h\left(W_{i}\right)\right) \\
& =E_{P_{2}}\left(f\left(W_{k}\right)\right) \cdot E_{P_{2}}\left(g\left(W_{j}\right)\right) \cdot E_{P_{2}}\left(h\left(W_{i}\right)\right)
\end{aligned}
$$

Now, since under $P_{2}$, the distribution of $U_{S_{r}\left(\omega_{1}\right)}\left(\omega_{2}\right)-U_{S_{r-1}\left(\omega_{1}\right)}\left(\omega_{2}\right)$ is the same as that of $U_{S_{r}\left(\omega_{1}\right)-S_{r-1}\left(\omega_{1}\right)}=U_{Z_{r}\left(\omega_{1}\right)}\left(\omega_{1}\right.$ fixed $)$, we have for each Borel positive function $\psi: \mathbf{R}_{+} \rightarrow \mathbf{R}$

$E_{P_{2}}\left(\psi\left(W_{r}\right)\right)=\int_{R_{+}} \psi\left(Z_{r}\left(\omega_{1}\right)-t\right) \mu_{Z_{r}\left(\omega_{1}\right)}(\mathrm{d} t), \quad r=i, j, k$

From lemma 3.11, the functions

$\omega_{1} \rightarrow \int_{R_{+}} \psi\left(Z_{r}\left(\omega_{1}\right)-t\right) \mu_{Z_{r}\left(\omega_{1}\right)}(\mathrm{d} t), r=i, j, k$, are Borel functions of the random variables $Z_{r}$, thus they are independent under the probability $P_{1}$. Therefore, applying $E_{P_{1}}$ to both sides of (3.14) we get (3.13).

As for the process $X_{n}$, the counterpart of proposition 3.9 is the following:

3.15. Proposition: If $f: R_{+} \rightarrow R$ is positive measurable and if $B \in \mathbf{B}_{R_{+}}$, then we have:

$$
E_{P}\left(f\left(X_{n}\right)\right)=\int_{\mathrm{R}_{+} \times \mathrm{R}_{+}} f(s-t) \mu_{s}(\mathrm{~d} t) \cdot \lambda^{* n}(\mathrm{~d} s)
$$




$$
\begin{gathered}
P\left(X_{n} \in B\right)=\int_{\mathrm{R}_{+}} \mu_{s}(s-B) \lambda^{* n}(\mathrm{~d} s) \\
E_{P}\left(X_{n}\right)=n \cdot(\alpha-\beta)
\end{gathered}
$$

For the proof, use the formula $X_{n}=S_{n}-U_{S_{n}}$ and routine integration.

3.16. Example: Let $0<c<1$ and let us take as measure $\mu_{s}$ the unit mass at the point $c s$, that is, the Dirac measure $\mu_{s}=\delta_{c s}, \quad s \in \mathrm{R}_{+}$. It easy to check that $\mu_{s+t}=\mu_{s} \times \mu_{t}$ for all $s, t$ in $\mathrm{R}_{+}$Then for every probability measure $\lambda$ on $\mathrm{R}_{+}$

we have: $P\left(U_{Z_{1}} \in B\right)=\int_{\mathrm{R}_{+}} \mu_{s}(B) \lambda(\mathrm{d} s)=\lambda\left(c^{-1} B\right)$. This gives the distribution of the release process in this case:

$$
P\left(U_{S_{n}} \in B\right)=\int_{\mathrm{R}_{+}} \mu_{s}(B) \lambda^{* n}(\mathrm{~d} s)=\lambda^{* n}\left(c^{-1} B\right) .
$$

Since we have $\lambda^{* n}\left(c^{-1} B\right)=P\left(c S_{n} \in B\right)$, we deduce that the release rule consists in removing from $S_{n}$ the quantity $c S_{n}$.

Likewise it is straightforward, from Proposition 3.14, that

$$
\begin{aligned}
& P\left(X_{n} \in B\right)=\int_{\mathrm{R}_{+}} \mu_{s}(s-B) \cdot \lambda^{* n}(\mathrm{~d} s) \\
& =\int_{\mathrm{R}_{+}} \delta_{c s}(s-B) \lambda^{* n}(\mathrm{~d} s) \\
& =\int_{\mathrm{R}_{+}} 1_{(1-c)^{-1} B}(s) \lambda^{* n}(\mathrm{~d} s) \\
& =\lambda^{* n}\left((1-c)^{-1} B\right)
\end{aligned}
$$

from which we deduce that the distribution of the storage process is

$$
P\left(X_{n} \in B\right)=P\left((1-c) S_{n} \in B\right) .
$$

One can give more examples in this way by choosing the distribution $\lambda$ or/and the semigoup $\left\{\mu_{x}, x \geq 0\right\}$. Consider the following simple example:

3.17. Example: Take $\lambda$ the $0-1$ Bernoulli distribution with probability of success $p$. In this case the semigroup $\left\{\mu_{x}, x \geq 0\right\}$ is a sequence $\mu_{n}$ of probabilities with $\mu_{n}$ supported by $\{1,2, \cdots, n\}$ for $n \geq 1$ and $\lambda^{* n}$ is the Binomial distribution. So we get from proposition 3.9

$$
\begin{aligned}
P\left(U_{S_{n}} \in B\right) & =\int_{\mathrm{R}_{+}} \mu_{s}(B) \lambda^{* n}(\mathrm{~d} s) \\
& =\sum_{k=0}^{n}\left(\begin{array}{l}
n \\
k
\end{array}\right) p^{k}(1-p)^{n-k} \mu_{k}(B)
\end{aligned}
$$

Likewise we get the distribution of $X_{n}$ from proposition $\mathbf{3 . 1 5}$ as :

$$
\begin{aligned}
& P\left(X_{n} \in B\right)=\int_{\mathrm{R}_{+}} \mu_{s}(s-B) \lambda^{* n}(\mathrm{~d} s) \\
& =\sum_{k=0}^{n}\left(\begin{array}{l}
n \\
k
\end{array}\right) p^{k}(1-p)^{n-k} \mu_{k}(s-B)
\end{aligned}
$$

\section{Limit Theorems}

Due to the simple structure of the processes $U_{S_{n}}$ and $X_{n}$ (Theorems 3.8, 3.12), it is not difficult to establish a SLLN and a CLT for them.

4.1. Theorem: For the storage process $X_{n}$ and the release rule process $U_{S_{n}}$, we have:

$$
\lim _{n} \frac{X_{n}}{n}=\alpha-\beta=E_{P}\left(X_{1}\right)
$$

and

$$
\lim _{n} \frac{U_{S_{n}}}{S_{n}}=\frac{\beta}{\alpha}
$$

Proof: Since $S_{n}$ and $U_{S_{n}}$ are simple random walks with $E_{P}\left(Z_{1}\right)=\alpha$ and $E_{P}\left(U_{S_{1}}\right)=\beta$, we have:

$\lim _{n} \frac{S_{n}}{n}=\alpha$ and $\lim _{n} \frac{U_{S_{n}}}{n}=\beta$, by the classical S.L.L.N.

So we deduce:

$$
\lim _{n} \frac{X_{n}}{n}=\lim _{n} \frac{S_{n}-U_{S_{n}}}{n}=\alpha-\beta
$$

and

$$
\lim _{n} \frac{U_{S_{n}}}{S_{n}}=\lim _{n} \frac{\frac{U_{S_{n}}}{n}}{\frac{S_{n}}{n}}=\frac{\beta}{\alpha} .
$$

4.2. Proposition: Under the conditions:

$$
\begin{aligned}
& \int_{\mathrm{R}_{+} \times \mathrm{R}_{+}} t^{2} \mu_{s}(\mathrm{~d} t) \cdot \lambda(\mathrm{d} s)<\infty \text { and } \\
& \int_{\mathrm{R}_{+} \times \mathrm{R}_{+}} s \cdot t \mu_{s}(\mathrm{~d} t) \cdot \lambda(\mathrm{d} s)<\infty \text {, the variances } \sigma_{U}^{2} \text { and } \sigma_{X_{1}}^{2}
\end{aligned}
$$
of the random variables $U_{Z}$ and $X_{1}$ are finite. The conditions can respectively be written as

$$
\int_{\mathrm{R}_{+}} E\left(U_{s}^{2}\right) \cdot \lambda(\mathrm{d} s)<\infty
$$

and

$$
\int_{\mathrm{R}_{+} \times \mathrm{R}_{+}} s \cdot E\left(U_{s}\right) \cdot \lambda(\mathrm{d} s)<\infty .
$$

Proof: We have $\sigma_{U}^{2}=\int_{\mathrm{R}_{+} \times \mathrm{R}_{+}} t^{2} \mu_{s}(\mathrm{~d} t) \cdot \lambda(\mathrm{d} s)-\beta^{2}$, so the first condition gives $\sigma_{U}^{2}<\infty$. On the other hand we have 


$$
\sigma_{X_{1}}^{2}=\int_{\mathrm{R}_{+} \times \mathrm{R}_{+}}(s-t)^{2} \mu_{s}(\mathrm{~d} t) \cdot \lambda(\mathrm{d} s)-(\alpha-\beta)^{2}
$$

and

$$
\begin{aligned}
& \int_{\mathrm{R}_{+} \times \mathrm{R}_{+}}(s-t)^{2} \mu_{s}(\mathrm{~d} t) \cdot \lambda(\mathrm{d} s) \\
= & \left.\int_{\mathrm{R}_{+} \times \mathrm{R}_{+}}\left(s^{2}+t^{2}\right) \mu_{s}(\mathrm{~d} t) \cdot \lambda(\mathrm{d} s)\right) \\
& -2 \int_{\mathrm{R}_{+} \times \mathrm{R}_{+}} s \cdot t \mu_{s}(\mathrm{~d} t) \cdot \lambda(\mathrm{d} s)
\end{aligned}
$$

Since the variance $\sigma^{2}$ of $Z_{n}$ is finite we have $\int_{\mathrm{R}_{+} \times \mathrm{R}_{+}} s^{2} \mu_{s}(\mathrm{~d} t) \cdot \lambda(\mathrm{d} s)=\int_{\mathrm{R}_{+}} s^{2} \cdot \lambda(\mathrm{d} s)<\infty$, so the conclusion follows.

Finally we get under the conditions of proposition 4.2:

4.3. Theorem: Assume the conditions of proposition 4.2. Then the normalized sequences of random variables:

$$
T_{n}=\frac{U_{S_{n}}-n \cdot \beta}{\sigma_{U} \sqrt{n}} \text { and } R_{n}=\frac{X_{n}-n \cdot(\alpha-\beta)}{\sigma_{X_{1}} \sqrt{n}}
$$

both converge in distribution to the Normal law $N(0,1)$.

Proof: The condition of the theorem insures the finiteness of the variances $\sigma_{U}^{2}$ and $\sigma_{X_{1}}^{2}$. Now the conclusion results from the fact that $U_{S_{n}}$ and $X_{n}$ are simple random walks and the Lindberg Central Limit Theorem. To see this, we use the method of characteristic functions. Let us denote by $f_{\theta}$ the characteristic function of the random variable $\theta$. Since by Theorem 3.8 the components $U_{S_{k}}-U_{S_{k-1}}$ of $U_{S_{n}}$ have the same distribution as $U_{Z_{1}}$, we have

$$
\begin{aligned}
& f_{T_{n}}(t)=\exp \left(\text { it } T_{n}\right) \\
& =\left(f_{U_{Z_{1}}-\beta}\left(\frac{t}{\sigma_{U} \sqrt{n}}\right)\right)^{n} \\
& =\left\{1+\frac{i^{2} \sigma_{U}{ }^{2}}{2}\left(\frac{t}{\sigma_{U} \sqrt{n}}\right)^{2}+o\left(\frac{|t|}{\sigma_{U} \sqrt{n}}\right)^{2}\right\}^{n} \\
& =\left\{1-\frac{t^{2}}{2 n}+o\left(\frac{t^{2}}{n}\right)\right\}^{n} \rightarrow \exp \left(-\frac{t^{2}}{2}\right)
\end{aligned}
$$

where the second equality comes from the Taylor expansion of $f_{U_{Z_{1}}-\beta}$. It is well known that this limit is the characteristic function of the random variable $N(0,1)$. The same proof works for $R_{n}$, using the components of the process $X_{n}$ as given in Theorem 3.12.

In some storage systems, the changes due to supply and release do not take place regularly in time. So it would be more realistic to consider the time parameter $n$ as random. We will do so in what follows and will consider the asymptotic distributions of the processes $U_{S_{n}}$, and $X_{n}$, when properly normalized and random- ized. First let us put for each $k$,

$$
\begin{gathered}
A_{k}=\frac{U_{S_{k}}-U_{S_{k-1}}-\beta}{\sigma_{U}}, \text { and } \\
B_{k}=\frac{Z_{k}-\left(U_{S_{k}}-U_{S_{k-1}}\right)-(\alpha-\beta)}{\sigma_{X_{1}}} .
\end{gathered}
$$

Then we have:

4.4. Theorem: Let $\left\{N_{n}: n \geq 1\right\}$ be a sequence of integral valued random variables, independent of the $A_{k}$ and $B_{k}$.

If $\frac{N_{n}}{n}$ converges in probability to 1 , as $n \rightarrow \infty$, then the randomized processes:

$$
\frac{\sum_{1}^{N_{n}} A_{k}}{\sqrt{n}} \text { and } \frac{\sum_{1}^{N_{n}} B_{k}}{\sqrt{n}}
$$

both converge in distribution to the Normal law $N(0,1)$.

Proof: It is a simple adaptation of [7], VIII.4, Theorem 4, p. 265.

\section{Conclusion}

In this paper, we presented a simple stochastic storage process $X_{n}$ with a random walk input $S_{n}$ and a natural release rule $U_{S_{n}}$. Realistic conditions are prescribed which make this process more tractable when compared to those models studied elsewhere (see Introduction). In particular the conditions led to a simple structure of random walk for the processes $U_{S_{n}}$ and $X_{n}$, which has given explicitly their distributions, and a rather good insight on their asymptotic behavior since a SLLN and a CLT has been easily established for each of them. Moreover, a slightly more general limit theorem has been obtained when time is adequately randomized and both processes $U_{S_{n}}$ and $X_{n}$ properly normalized.

\section{Acknowledgements}

I gratefully would like to thank the Referee for his appropriate comments which help to improve the paper.

\section{REFERENCES}

[1] E. Cinlar and M. Pinsky, "On Dams with Additive Inputs and a General Release Rule," Journal of Applied Probability, Vol. 9, No. 2, 1972, pp. 422-429. doi: $10.2307 / 3212811$

[2] E. Cinlar and M. Pinsky, "A Stochastic Integral in Storage Theory," Probability Theory and Related Fields, Vol. 17, No. 3, 1971, pp. 227-240. doi:10.1007/BF00536759

[3] J. M. Harrison and S. I. Resnick, "The Stationary Distribution and First Exit Probabilities of a Storage Process with General Release Rule," Mathematics of 
Operations Research, Vol. 1, No. 4, 1976, pp. 347-358. doi:10.1287/moor.1.4.347

[4] J. M. Harrison and S. I. Resnick, "The Recurrence Classification of Risk and Storage Processes," Mathematics of Operations Research, Vol. 3, No. 1, 1978, pp. 57-66. doi:10.1287/moor.3.1.57

[5] K. Yamada, "Diffusion Approximations for Storage Pro- cesses with General Release Rules," Mathematics of Operations Research, Vol. 9, No. 3, 1984, pp. 459-470. doi:10.1287/moor.9.3.459

[6] P. A. Meyer, "Probability and Potential," Hermann, Paris, 1975.

[7] W. Feller, "An Introduction to Probability Theory and Its Applications," 2nd Edition, Wiley, Hoboken, 1970. 